\title{
Positive Psychologists on Positive Psychology: Barbara Fredrickson
}

Interview by

Aaron Jarden

\begin{abstract}
Barbara Fredrickson is the Kenan Distinguished Professor of Psychology and Director of the Positive Emotions and Psychophysiology Lab (PEP Lab) at the University of North Carolina, and is a leading scholar within positive psychology, social psychology, and affective science. Barbara's research centers on positive emotions and human flourishing, and her work is cited widely, including her 2009 book, Positivity, which describes the relevance of her 20-year research programme on positive emotions for a general readership, and her Broaden-and-Build Theory of positive emotions.
\end{abstract}

In general terms and in your mind, what are some of the distinctive features of positive psychology?

The distinctive features of positive psychology are rather nebulous because a lot of things that can be called positive psychology are often not necessarily termed positive psychology. At the overarching general level would be looking at the aspects of human nature that help us become a better version of ourselves over time, that help propel us towards growth, or greater confidence, greater resilience, greater honesty, greater integrity. The study of those aspects and a lot of that work happens under the umbrella of positive psychology, and a lot of it happens outside of it. The boundaries I think are very blurry.

\section{What are some things that positive psychology has achieved to date?}

One of the biggest achievements of positive psychology to date is just getting on the public radar; of being clearly an area of science worth investing in, worth paying attention to, worth applying. The tremendous interest right now in creating resilience training programs, and trying to create a more resilient public, or a more resilient military force, really speaks to the strength of this perspective.

\section{If you had a magic wand, what would be one thing that you would change about positive psychology?}

That there would be a clear source of funding for positive psychology. Right now it's unclear where more funding for the more basic science pieces of it will come from. The demand for the fruits of positive psychology is far larger than the supply of good science, and that in part is because we don't necessarily have the funds to be training people in the basic science areas of positive psychology. Positive psychology spans both basic science and applied science, and right now we have a situation where the applied side is not necessarily being fed by enough of 
the basic science pieces. That's in part because funding for the basic science pieces is harder to come by and training dollars for training scientists in those areas is tough to come by. So that would be my magic wand: to invest in the basic science side, especially in training the next generation of people to do it.

\section{What do you think is the new hot topic for positive psychology over the next few years?}

Health is getting stronger attention in that we need to go beyond looking at wellbeing or happiness as simply judgments and emotions, and really anchor them in how they are affecting the bottom line of people's life expectancy, their illness histories, the dollars they cost their employers in health care visits, and also more and more in how much positive psychology within organizations affects their bottom line. I think ways of making those metrics concrete are especially vital. And then at the basic science level, looking at how wellbeing and emotions are part of larger dynamic systems is going to be ever more crucial because wellbeing is not a single state, you know-it's not like you reach some happiness plateau and you're forever there-instead people get caught up in upward spirals or downward spirals, or some selfsustaining system in one way or another. We need to better understand those temporal dynamics and how they unfold and how they reinforce wellness or illness over time.

\section{Can you tell me a little bit about your work in the area of positive psychology?}

I work on emotions, and positive emotions in particular. My students and collaborators and I are interested in everything about positive emotions, which are short-lived, momentary, fleeting experiences that have, in the moment, ways of altering the ways we think, the ways we behave, the way we see other people. Over time those momentary effects of positive emotions add up and really change who we become, change how people grow and change over time. So I'm interested in those short-term and long-term accumulated effects of positive emotions. We're looking at those in a wide variety of ways, especially right now focusing on physical health.

\section{As you have been working in the field of positive psychology, what's one learning experience that's made a big difference for you?}

One that's really a challenge is going from basic research on laboratory studies and finding out the cognitive effects, say, of positive emotions, to making the transition into applied work, and trying to help people achieve more positive emotions so they can have the good outcomes that those emotions may bring. There is a vexing additional piece of people's wishful thinking: people sometimes so hunger to have wellbeing and have positive emotions that they lose sight of whether they are really having them when they fill out a questionnaire. There's a lot of, in jargon terms, experimenter demand or demand characteristics, but it's just, in general terms, kind of wishful thinking. So as a researcher, working in that more applied domain, you really need to be looking to see whether this is a genuine, bona fide, positive emotion that this person is reporting, or do they wish they were feeling a positive emotion? Do they earnestly wish that, so they're in a way presenting a counterfeit positive emotion? Because wellbeing and wellness and happiness are all things that people so earnestly strive for, they can sometimes lose sight of 'What's the real thing?' versus just too quickly saying, 'Oh yes, I'm happy', without really examining the data for that. 


\section{What's your proudest moment in the field of positive psychology to date?}

It's when people put the ideas into use. I'm trained as a lab researcher and a basic scientist. I think of myself as an emotion theorist. What positive psychology offers really well is an interface between people doing the basic science, and practitioners in all different kinds of domains, industry, education, the military and so on, who want to put that science into action. So some of the proudest moments I've had are when people do that, they translate what I've been working on into application, and are finding success with that. So again, there is only so much one person can do, and the ideas that emerge from the science that I've been doing, I can communicate those in a way that I can pass the baton on to others who can do great things with those ideas. That's something that makes me really proud.

\section{Who are the emerging and unknown researchers in positive psychology to look out for?}

I can give some names of people I know well because they have been junior collaborators of mine. Sarah Algoe is one who is doing phenomenal work on gratitude in particular, and other ways that certain positive emotional moments can transform social opportunities and relationships. She's working at the interface of emotions and relationships. One of my current doctoral students, Bethany Kok, is diving into the physiological substrates of the upward spirals that we think sustain people's wellbeing, so she's a name to keep an eye out for in the future.

\section{What's one book, other than your book Positivity, that you think all those getting into the field and learning about positive psychology should read?}

The best initial take is perhaps Sonja Lyubomirsky's book The How of Happiness, which is excellent in helping people understand the basic exercises that have a track record for improving wellbeing, and in helping people understand the hurdles and the sluggishness they must overcome, for happiness and wellbeing to improve. I think it gives a good realistic account.

What's one piece of advice for aspiring positive psychology researchers and or practitioners?

Stay actively invested in the positive psychology community and also in the home discipline your training is in, whether it's social psychology or affective neuroscience or others. Both of those affiliations are really vital. Identifying in positive psychology can be useful and productive, but it wouldn't be the only identity that you would want or the only identity hat that you would want to wear. Make sure that the work that you are doing is equally turning heads within a related discipline that isn't necessarily under the umbrella of positive psychology.

\section{Author}

Aaron Jarden

Open Polytechnic of New Zealand

aaron.jarden@openpolytechnic.ac.nz 